\title{
Power Efficient Optimal Structure CAM-Cell in QCA Technology
}

\author{
Ali H. Majeed ${ }^{1,2^{*}}$, Bassam A. Salih ${ }^{1}$, Mohd Shamian Bin Zainal ${ }^{2}$ and Danial M.D. Nor ${ }^{2}$ \\ 'Electrical Department, Faculty of Engineering, University of Kufa, Kufa, Iraq; alih.alasady@uokufa.edu.iq, \\ bassama.albaidhani@uokufa.edu.iq \\ ${ }^{2}$ Faculty of Electrical and Electronic Engineering, Universiti Tun Hussein Onn Malaysia, Johor, Malaysia; \\ Shamian@uthm.edu.my, Danial@uthm.edu.my
}

\begin{abstract}
Background/Objectives: Quantum-dot Cellular Automata (QCA) is a new nanoscale technology that is expected to be a persuasive replacement of CMOS technology. QCA technology competing for features concerning low power consumption, small physical size, and ultra-high speed. These features are beneficial for memory design. This study presents a new structure of the CAM cell in QCA technology. The presented cell is carried out in an optimal form by using a proposed unique block. Method: In this study, the QCADesigner tool was used for circuit design and the QCAPro tool was used for power analysis. Both of these tools were used in default parameters. Findings: It was found that the proposed CAM cell is less complexity and efficient in power consumption with improvements by $6 \%, 29 \%$ and $15 \%$ in terms of leakage, switching and total energy consumption. Improvement: The CAM cell is the main building block for the whole memory. So, the reduction in complexity and power consumption in the CAM cells is important to reach an optimal memory circuit.
\end{abstract}

Keywords: CAM Cell, Memory Cell, Nanoelectronics, QCA Technology, Quantum-dot Cellular Automata

\section{Introduction}

Quantum-dot Cellular Automata (QCA) is a new technology that researchers have presented as a solution to overcome and minimize some CMOS limitations concerning the consumption of power, leakage current, and physical size ${ }^{1,2}$. QCA is a recent nanoscale technology with design techniques that can be considered as a good replacement for those used in classical circuit design. It can achieve good performance with very little consumed power compared to CMOS technology ${ }^{3}$. The main building block in QCA design is a square cell that has four holes (dots) with two electrons. When Coulomb repulsion occurs, the two electrons occupy holes diagonally inside the cell 1,5 . QCA memory cell design has been attracted by many researchers. Research efforts $\frac{6-11}{}$ have focused on the design and performance of the Random Access Memory cell (RAM cell) in QCA. In this work, a new Content
Addressable Memory cell (CAM cell) has been proposed. The CAM, which differs from RAM, is a kind of memory that is likely used in applications that require searching with very high speed ${ }^{12}$. In CAM, the access time to stored data in memory is reduced ${ }^{4}$. A new efficient CAM cell with lower complexity (cell counts) and minimum power required has been presented. The gates used to design this structure are XOR gate with two inputs and a majority gate of three inputs. A QCA Designer simulation tool was used for modeling and performance evaluation of the proposed CAM cell structure.

The contents of this study will be as follows: Section 2 will present a review of QCA technology and CAM cell. The proposed model of CAM cell will introduce in Section 3. Section 4 will provide the results and compression with discussion. The conclusion will summarize the work in Section 5.

*Author for correspondence 


\section{Preliminaries}

\subsection{QCA Technology}

QCA is a new nanoscale technology that was introduced for the first time by ${ }^{13}$. The basic building unit in QCA circuits is a square cell. Each cell contains four holes with two electrons. The Coulombic force controls the electron's movement inside the cell ${ }^{14}$. Logic states whether 0 or 1 in the cell depends on the electrons configuration inside the cell. Cell polarization can be calculated using Equation (1). Where pi represents the probability of the electron existing inside dot i.The cell represents logic 1 if it is polarized equal to +1 and if it is polarized equal to -1 it represents logic $0^{\underline{10}}$. Figure 1 explain the QCA cell structure with its polarization.

$$
p=\left(\frac{p 1-p 2+p 3-p 4}{p 1+p 2+p 3+p 4}\right)
$$

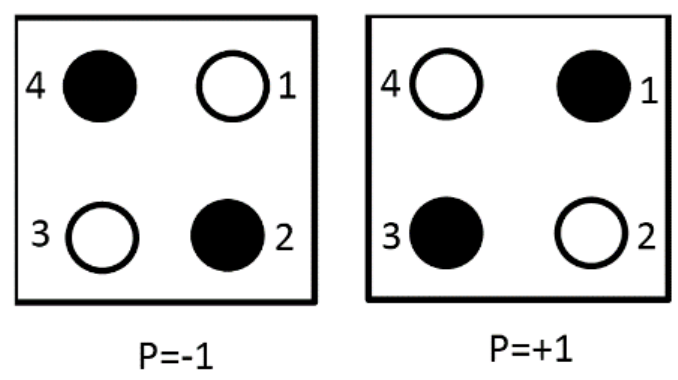

Figure 1. Polarized QCA Cell forms.

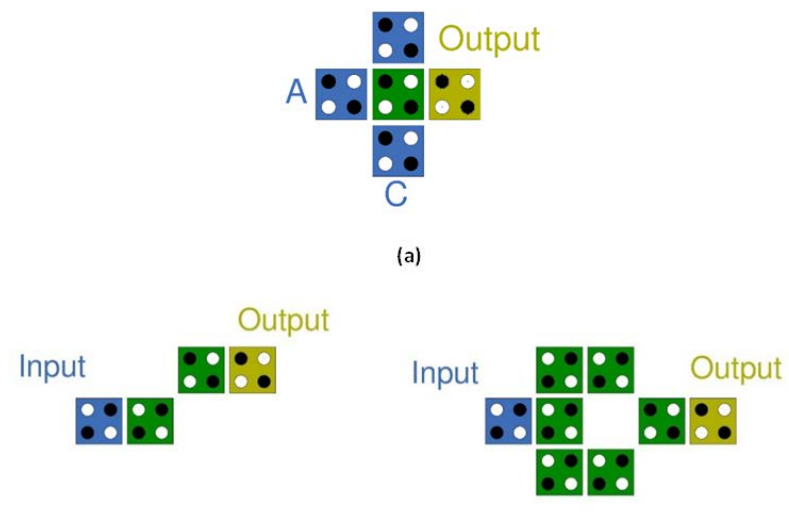

(b)

Figure 2. QCA Basic gates. (a) 3-inputs Majority structure. (b) Inverter structures.

\subsubsection{QCA Basic Gates}

The basic gates in QCA technology are the majority gate and the inverter as shown in Figure $2^{\frac{15}{}}$. The majority gate function for 3 inputs $(A, B, C)$ is $A B+A C+B C$. OR or AND gates with 2-inputs can be constructed utilizing 3 -inputs majority gate. This can be done by connecting any of the three bits of majority gate to logic 1 or logic 0 . Many papers were published in literature focused on the majority gate for both three and five bits $\frac{16-23}{}$.

\subsubsection{Clock Signal}

In QCA circuits, a clocking signal is necessary for controlling the information flow ${ }^{24}$. The circuit can be divided into four different regions (zones). Each zone has four phases. The clock signal controls the barrier between the holes to allow or prevent electron tunneling 25 . Figure 3 shows the four-zone clocking scheme.

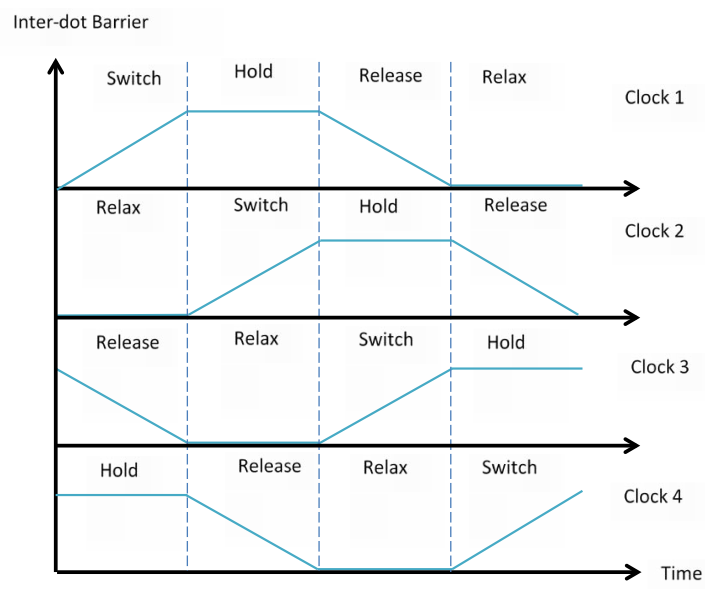

Figure 3. The common clock zones in QCA.

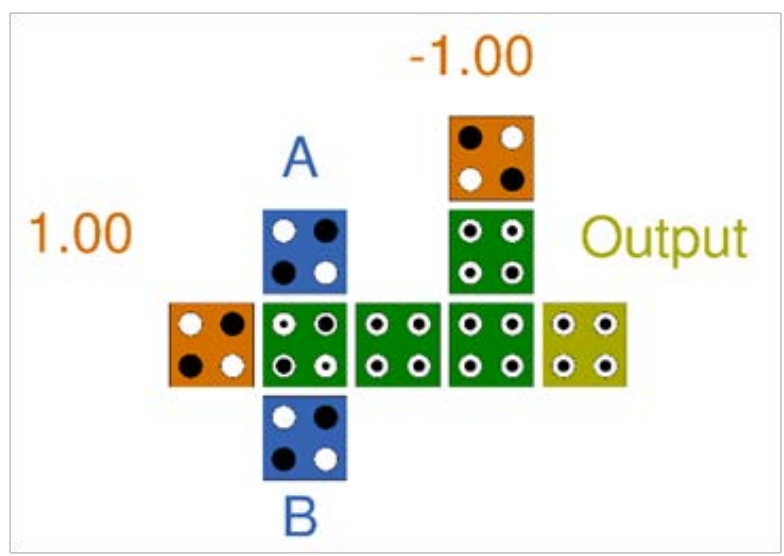

Figure 4. Optimal QCA XOR gate ${ }^{33}$. 


\subsubsection{The XOR Gate}

The 2-input XOR gate is widely used in logic circuits. In QCA, researchers introduced many forms of 2-input XOR gates $\frac{26-33}{}$. Some of them follow Boolean function while others use the inherent capability of QCA to get the output. The optimal design XOR layout was presented by ${ }^{33}$ as shown in Figure 4. This layout will be utilized in this study to obtain the XNOR gate by exchanging the fixed cells.

\subsection{CAM}

CAM structure is built for very fast memory searching purposes. The CAM has the ability to search all of its contents within just one clock cycle. To access stored data on CAM, it makes a searching process, produces a match signal and then, restores addresses of the content.

\section{The Proposed Structure}

This section will explaina new CAM cell structure formative by a proposed unique block.

\subsection{The Proposed Unique Block}

As mentioned before, the most significant challenge for researchers is to minimize cell count and to increase speed. This research introducesa new QCA blockas attempting to improve the performance of CAM memory. Equation (2) describes the function of this block. An XNOR and majority gates were utilized to design the unique block as shown in Figure 5.

Output $=(\mathrm{A} \odot \mathrm{F})+\mathrm{K}$

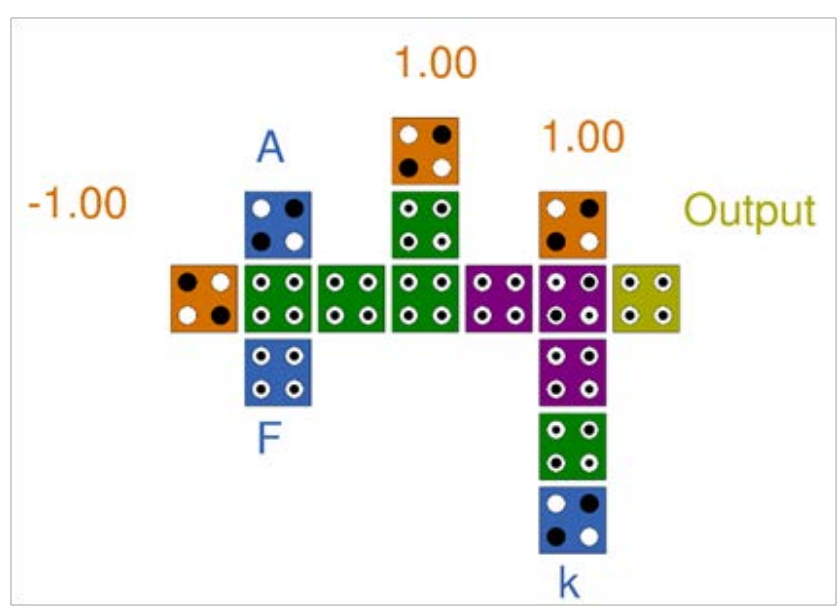

Figure 5. The proposed unique block.

\subsection{The Proposed CAM Cell}

Figure 6 shows the proposed QCA structure of the CAM cell. The structure of this cell consists of a single unique block and three of 3-input majority gates. The CAM cell comprised of two main parts which are the proposed block and memory unit. The memory unit receives two signals which are Read/Write $\left(\mathrm{R} / \mathrm{W}^{\dagger}\right)$ control signal, and input data (I). Additionally, it has one output signal (F). The proposed block has three inputs which are input $(\mathrm{F})$ which comes from the memory unit, input (A) and input (K). It also has an output $(\mathrm{M})$ which determines its state according to $(\mathrm{A})$ and $(\mathrm{K})$ inputs. The operation of the memory unit depends on the value of the $\left(\mathrm{R} / \mathrm{W}^{\prime}\right)$ control signal. Connecting $(\mathrm{R} / \mathrm{W})$ line to logic ' 0 ' makes the output (F) tends to logic ' 0 ', which represents the (Write) operation whereas setting $\left(R / W^{\prime}\right)$ to logic ' 1 ', the memory cell will perform the (Read) operation accordingly. The memory unit functionality table is illustrated in Table 1. In the proposed unique block, the setting input $(\mathrm{K})$ to logic (1) will tend output (M) to (1), regardless of the logic state of $(\mathrm{A})$ and $(\mathrm{F})$. While connecting input $(\mathrm{K})$ to logic (0), the output (M) will be dependent on (A) and (F) values.

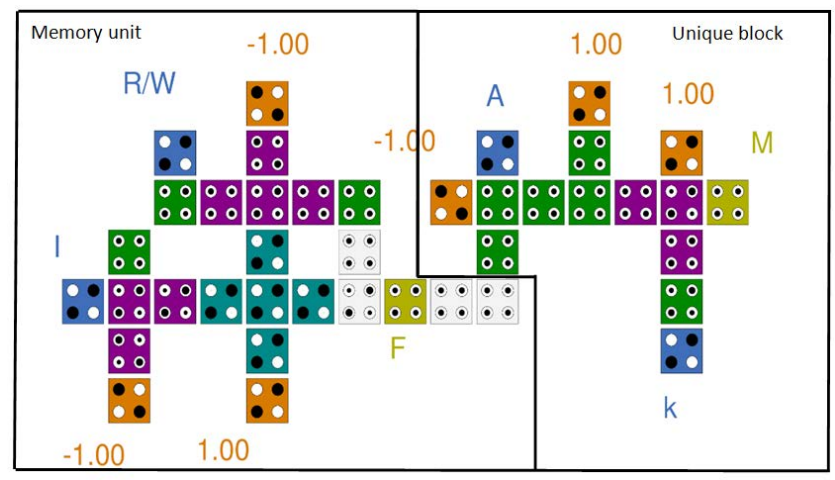

Figure 6. QCA layout of proposed CAM cell.

\section{Simulation Waveforms and Discussion}

The circuits simulation using QCA Designer software ${ }^{34}$ are provided in this section. The software parameters have been set to default. Figure 7(a) shows the simulation result of the proposed block while Figure 7(b) shows the simulation result of the proposed CAM cell as well. The CAM cell comparison table is given in Table (2).In addition, QCAPro software hyperlink ${ }^{35}$ has been used for power consumption analyses of the presented QCA 
circuits. The energy dissipation thermal map in the proposed CAM cell is shown in Figure 8. The analysis of power consumption is illustrated in Figure 9.

Table 1. Memory operation truth table

\begin{tabular}{|l|l|l|l|l|}
\hline Operation type & R/W & Previous F & I & F \\
\hline Write & 0 & $\mathrm{x}$ & 1 & 1 \\
\hline Write & 0 & $\mathrm{x}$ & 0 & 0 \\
\hline Read & 1 & 1 & $\mathrm{x}$ & 1 \\
\hline Read & 1 & 0 & $\mathrm{x}$ & 0 \\
\hline
\end{tabular}

(a)

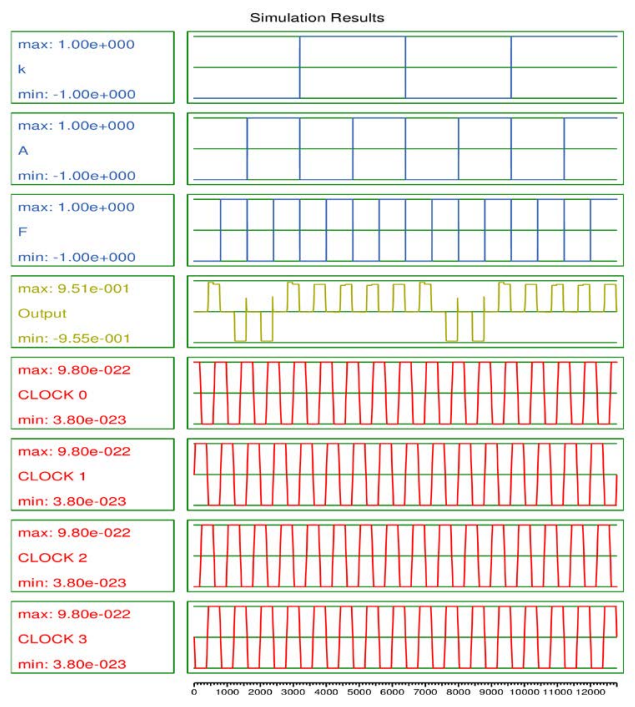

Figure 8. The energy dissipation thermal map for proposed CAM cell.

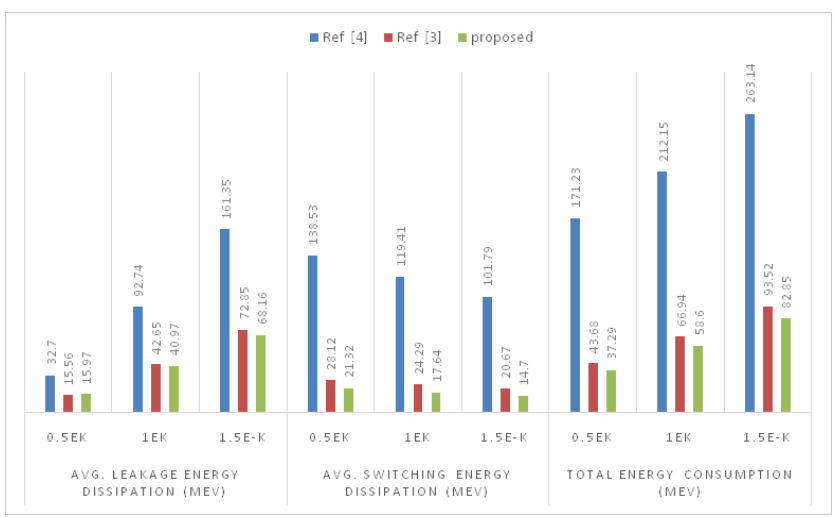

Figure 9. Power dissipation comparison of CAM Cell.

Table 2. CAM cell compression table

(b)

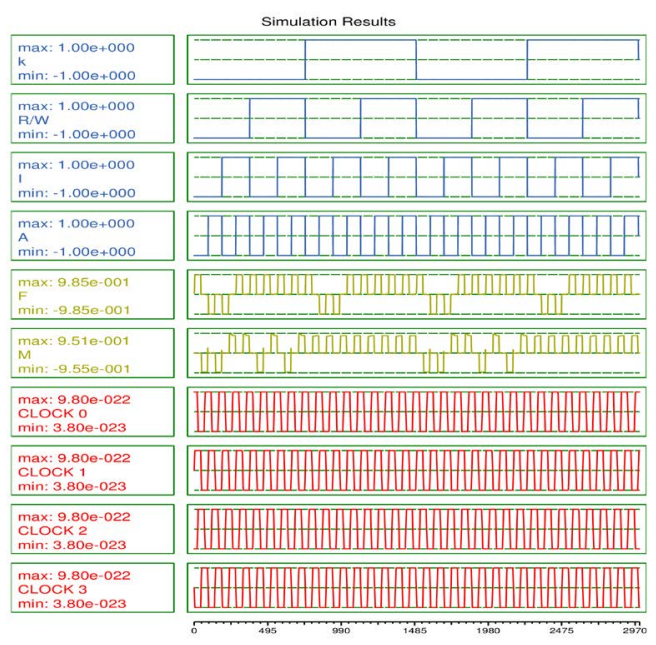

Figuire 7. Simulation output for. (a) Proposed block. (b) Proposed CAM cell.

\begin{tabular}{|l|l|l|l|}
\hline CAM cell & Cell counts & Delay & Area $(\mu \mathrm{m} 2)$ \\
\hline $\operatorname{In}^{4,12,3}$ & 100 & 2 & 0.14 \\
\hline $\operatorname{In}^{12}$ & 94 & 2 & 0.11 \\
\hline $\mathrm{In}^{3}$ & 46 & 1.25 & 0.04 \\
\hline proposed & 40 & 1.25 & 0.04 \\
\hline
\end{tabular}

As previously seen, the power analysis indicates that the proposed CAM cell is efficient in power consumption and makes improvements by $6 \%, 29 \%$ and $15 \%$ in terms of leakage, switching and total energy consumption. The results have been analyzed at three stages $\left(0.5 \mathrm{E}_{\mathrm{k}}, 1 \mathrm{E}_{\mathrm{k}}\right.$, and $1.5 \mathrm{E}_{\mathrm{k}}$ ).

\section{Conclusion}

In this research, a unique block with minimum cell counts was proposed for utilizing in CAM cell design. Next, a 
new power-efficient structure of CAM cell has been proposed. QCADesigner tool was used to design the circuits while the QCAPro tool was used for power analysis. Simulation results clearly showed through comparison that the proposed design performance is better than the same counterparts.

\section{References}

1. More than Moore: Creating High Value Micro/ Nanoelectronics Systems. Available from: https://www. springer.com/gp/book/9780387755922

2. Alkaldy E, Navi K. Reliability study of single stage multiinput majority function for QCA. International Journal of Computer Applications. 2013; 83(2):19-23. https://doi. org/10.5120/14420-2545

3. Sadoghifar A, Heikalabad SR. A Content-Addressable Memory structure using quantum cells in nanotechnology with energy dissipation analysis. Physica B: Condensed Matter. 2018; 537:202-6. https://doi.org/10.1016/j. physb.2018.02.024

4. Heikalabad SR, Navin AH, Hosseinzadeh M. Content addressable memory cell in quantum-dot cellular automata. Microelectronic Engineering. 2016; 163:140-50. https://doi. org/10.1016/j.mee.2016.06.009

5. Sherizadeh R, Navimipour NJ. Designing a 2-to-4 decoder on nanoscale based on quantum-dot cellular automata for energy dissipation improving. Optik. 2018; 158:477-89. https://doi.org/10.1016/j.ijleo.2017.12.055

6. Shamsabadi A, Ghahfarokhi BS, Zamanifar K, Movahedinia N. Applying inherent capabilities of quantum-dot cellular automata to design: D flip-flop case study. Journal of Systems Architecture. 2009; 55:180-7. https://doi. org/10.1016/j.sysarc.2008.11.001

7. Dehkordi MA, Shamsabadi AS, Ghahfarokhi BS, Vafaei A. Novel RAM cell designs based on inherent capabilities of quantum-dot cellular automata. Microelectronics Journal. 2011; 42:701-8. https://doi.org/10.1016/j.mejo.2011.02.006

8. Hashemi S, Navi K. New robust QCA D flip flop and memory structures. Microelectronics Journal. 2012; 43:929-40. https://doi.org/10.1016/j.mejo.2012.10.007

9. Angizi S, Sarmadi S, Sayedsalehi S, Navi K. Design and evaluation of new majority gate-based RAM cell in quantum-dot cellular automata. Microelectronics Journal. 2015; 46:43-51. https://doi.org/10.1016/j.mejo.2014.10.003

10. Khosroshahy MB, Moaiyeri MH, Navi K, Bagherzadeh N. An energy and cost efficient majority-based RAM cell in quantum-dot cellular automata. Results in Physics. 2017; 7:3543-51. https://doi.org/10.1016/j.rinp.2017.08.067

11. Abutaleb MM. A novel configurable flip flop design using inherent capabilities of quantum-dot cellular automata
Microprocessors and Microsystems. 2018; 56:101-12 https://doi.org/10.1016/j.micpro.2017.11.003

12. Khosroshahy MB, Moaiyeri $\mathrm{MH}$, Navi K. Design and evaluation of a 5-input majority gate-based contentaddressable memory cell in quantum-dot cellular automata. International Symposium on Computer Architecture and Digital Systems (CADS); 2017. p. 1-5. https://doi. org/10.1109/CADS.2017.8310671

13. Lent CS. Quantum cellular automata. Nanotechnology. 1993; 4:49-57. https://doi.org/10.1088/0957-4484/4/1/004

14. Qiu KM, Xia YS. Quantum-dots cellular automata comparator. 2007 7th International Conference on ASIC; 2007. p. 1297-300.

15. SeyediS, Navimipour NJ. An optimized design of full adder based on nanoscale quantum-dot cellular automata. Optik. 2018; 158:243-56. https://doi.org/10.1016/j. ijleo.2017.12.062

16. Rumi Z, Walus K, Wei W, Jullien GA. A method of majority logic reduction for quantum cellular automata. IEEE Transactions on Nanotechnology. 2004; 3:443-50. https:// doi.org/10.1109/TNANO.2004.834177

17. Rui Z, Gupta P, Jha NK. Synthesis of majority and minority networks and its applications to QCA, TPL and SET based nanotechnologies. 18th International Conference on VLSI Design held jointly with 4th International Conference on Embedded Systems Design; 2005. p. 229-34.

18. Zhang R, Jha NK. Threshold/majority logic synthesis and concurrent error detection targeting nanoelectronic implementations. Proceedings of the 16th ACM Great Lakes symposium on VLSI; 2006. p. 8-13. https://doi. org/10.1145/1127908.1127913 PMCid:PMC2792020

19. Navi K, Farazkish R. New efficient five-input majority gate for quantum-dot cellular automata. Journal of Nanoparticle Research. 2012; 1(14):1252. https://doi.org/10.1007/ s11051-012-1252-3

20. Tehrani MA, Navi K, Kia-kojoori A. Multi-output majority gate-based design optimization by using evolutionary algorithm.SwarmandEvolutionaryComputation.2013;10:25-30. https://doi.org/10.1016/j.swevo.2012.12.002

21. Sen B, Sahu Y, Mukherjee R, Nath RK, Sikdar BK. On the reliability of majority logic structure in quantum-dot cellular automata. Microelectronics Journal. 2016; 47:7-18. https://doi.org/10.1016/j.mejo.2015.11.002

22. Hosseinzadeh $\mathrm{H}$, Heikalabad SR. A novel fault tolerant majority gate in quantum-dot cellular automata to create a revolution in design of fault tolerant nanostructures, with physical verification. Microelectronic Engineering. 2018; 192:52-60. https://doi.org/10.1016/j.mee.2018.01.019

23. A new 5-input Majority Gate Without Adjacent Inputs Crosstalk Effect in QCA Technology. Available from: https://www.researchgate.net/publication/331453919_A_ 
new_5-input_Majority_Gate_Without_Adjacent_Inputs_ Crosstalk_Effect_in_QCA_Technology

24. Circuit Design with Multi-input Majority Function for QCA and Nanotechnologies. Available from: https://www. researchgate.net/publication/275354194_Digital_circuits_Design_with_Multi-input_Majority_function_for_ Nanotechnology

25. Quantumdot Cellular Automata of Flip Flops. Available from: https://pdfs.semanticscholar.org/0b23/41b5a206d1e c5fb26f73433ceaa035cd6ba3.pdf

26. Beigh MR, Mustafa M, Ahmad F. Performance evaluation of efficient XOR structures in Quantum-Dot Cellular Automata (QCA). Circuits and Systems. 2013; 4:147-56. https://doi.org/10.4236/cs.2013.42020 A new F-shaped XOR gate and its implementations as novel adder circuits based Quantum-dot cellular Automata (QCA). Available from: https://www.semanticscholar.org/paper/A-new-F-shapedXOR-gate-and-its-implementations-as-Ahmad-Ahmad/90 5a2c5f2a9b7752cf7ef943f64cfd3d81114915

27. Angizi S, Alkaldy E, Bagherzadeh N, Navi K. Novel robust single layer wire crossing approach for exclusive OR sum of products logic design with quantum-dot cellular automata. Journal of Low Power Electronics. 2014; 10:259-71. https://doi.org/10.1166/jolpe.2014.1320

28. Chabi AM, Sayedsalehi S, Angizi S, Navi K. Efficient QCA exclusive-or and multiplexer circuits based on a nanoelectronic-compatible designing approach. International Scholarly Research Notices; 2014. p. 463967. https://doi.org/10.1155/2014/463967 PMid:27379276 PMCid:PMC4897480
29. Waje MG, Dakhole PK. Design and simulation of new XOR gate and code converters using Quantum Dot Cellular Automata with reduced number of wire crossings. 2014 International Conference on Circuits, Power and Computing Technologies [ICCPCT-2014]; 2014. p. 124550. https://doi.org/10.1109/ICCPCT.2014.7054942

30. Ajitha D, Ramanaiah KV, Sumalatha V. An efficient design of XOR gate and its applications using QCA. I-manager's Journal on Electronics Engineering. 2015; 5:22-9. https:// doi.org/10.26634/jele.5.3.3394

31. Chabi AM, Roohi A, Khademolhosseini H, Sheikhfaal S, Angizi S, Navi K. Towards ultra-efficient QCA reversible circuits. Microprocessors and Microsystems. 2017; 49:127-38. https://doi.org/10.1016/j.micpro.2016.09.015

32. Chen H, Lv H, Zhang Z, Cheng X, Xie G. Design and analysis of a novel low-power exclusive-OR gate based on quantum-dot cellular automata. Journal of Circuits, Systems and Computers. 2019; 19501-19541. https://doi.org/10.1142/S021812661950141X

33. Walus K, Dysart TJ, Jullien GA, Budiman RA. QCA Designer: a rapid design and simulation tool for quantum-dot cellular automata. IEEE Transactions on Nanotechnology. 2004; 3:26-31. https://doi.org/10.1109/ TNANO.2003.820815

34. Srivastava S, Asthana A,Bhanja S, Sarkar S. QCAPro- An error-power estimation tool for QCA circuit design. 2011 IEEE International Symposium of Circuits and Systems (ISCAS); 2011. p. 2377-80. https://doi.org/10.1109/ ISCAS.2011.5938081 